\title{
Resonant tunneling and localized states in a graphene monolayer with a mass gap
}

\author{
V. Zalipaev \\ Department of Mathematical Sciences, Loughborough University, Leicestershire, LE11 3TU, United Kingdom \\ and Krylov Institute, St. Petersburg, Russia \\ C. M. Linton \\ Department of Mathematical Sciences, Loughborough University, Leicestershire, LE11 3TU, United Kingdom \\ M. D. Croitoru \\ Physics Department, Antwerpen Universiteit, Antwerpen 2020, Belgium
}

A. Vagov

Institut für Theoretische Physik III, Bayreuth Universität, Bayreuth 95440, Germany

(Received 9 May 2014; published 5 February 2015)

\begin{abstract}
We study tunneling of quasiparticles through potential barriers in a graphene monolayer with the mass gap using a semiclassical (WKB) approach. The main equations are derived in a way similar to the WKB theory for the Schrödinger equation, which allows for explicit solutions at all orders. The analog of the classical action is used to distinguish types of possible stationary states in the system. The analysis focuses on the resonant scattering and the hole states localized in the vicinity of a barrier that are often overlooked. The scattering coefficients for the physically interesting limits are obtained by matching the WKB approximation with the known solutions at turning points. The localized states demonstrate unconventional properties and lead to alterations of the single particle density of states.
\end{abstract}

DOI: 10.1103/PhysRevB.91.085405

PACS number(s): 73.22.Pr, 72.80.Vp, 03.65.Sq

\section{INTRODUCTION}

Analysis of single particle scattering is an essential step in describing transport properties of graphene, where the interaction between carriers is typically small and the system is often in the ballistic regime [1-3]. The problem of a single particle tunneling for the Dirac-like Hamiltonian of graphene leads to many nontrivial properties, not observed in standard quantum mechanics. In particular it gives rise to the Klein tunneling [4], also known as Landau-Zenner tunneling in the theory of semiconductors [4-6], related to transmutation of electrons into holes and vice versa in the scattering process. This type of tunneling can be traced in the conductance or short-noise measurements in ballistic samples [7,8]. In experiments potential barriers are constructed by inserting charge impurities [9-11] or by creating terrace steps in the substrate [12]. Tunable barriers are achieved by varying the gate voltage [13]. This allows detailed studies of the effect of barrier shape on the transport [13] and paves the way for the creation of graphene-based devices [14].

The Achilles heel of graphene application in electronic devices is a vanishing gap in its spectrum. In order to open the gap and, furthermore, to control its value various methods have been proposed. For example, one can apply electrostatic gates [15] or create a superlattice [16]. Recent efforts in material science focused on designing new types of materials by doping graphene $[17,18]$ and by using boron nitride as a support of graphene sheets [19-21]. A chemical boron/nitrogen doping [18] opens the way for mass production of the graphene-based field transistors. Obviously carrier scattering and subsequently electric transport in graphenebased switching devices depends on the spectral gap, so that the latter has to be taken into account in the theoretical modeling.
Theory description of scattering is most trivial for rectangular or steplike barrier shapes [22-24]. Despite its simplicity this model is quite useful to illustrate general properties of the Klein-Landau-Zenner tunneling in graphene. However, it cannot capture many physical aspects of the tunneling and is certainly not valid for most experiments, where barriers are typically smooth. For smooth potential profiles the scattering problem is more complicated and significant efforts have been applied to study its various limiting cases [25-30]. When electrons hit an adiabatically slowly varying potential step the Klein tunneling can exhibit full particle transmission, when scattered in the direction normal to the barrier, or exponentially small transmission, when particles approach the barrier at an angle [25]. Klein tunneling through the barrier with a single maximum can be of a resonant character due to the presence of metastable or evanescent hole states inside the barrier [28-31], which then acts in a manner to a Fabry-Perot resonator. Analysis of resonant scattering can be done on a simplified model of a trapezoidal barrier, which has the advantage of being exactly solvable [31]. In a more general case of an arbitrary barrier semiclassical methods can be employed to obtain scattering coefficients as functions of the integral characteristics of the potential that cover many limiting cases [29,30]. Due to the matrix character of the Dirac equation a derivation of the semiclassical solution can proceed using multiple recipes that are formally different from the conventional WKB method used in quantum mechanics, e.g., using separate Hamiltonians for electron and hole states [29]. A more traditional approach, based on the expansion of the wave function in powers of the Planck constant $\hbar$, is also possible, being easier technically and arguably more intuitive physically [30]. The analog of classical trajectories 
that appear from the WKB solution of matrix equations, offers a convenient tool for classification of all scattering states associated with the barrier. In particular, it has been used to distinguish fully localized states [30] that are often overlooked in studies of the barrier scattering. An appearance of such states has been discussed earlier only for the rectangular barrier structure that, however, does not lead to a resonating Fabry-Perot structure [32].

In this work, having in mind recently obtained graphenebased devices with spectral gap [18,21], we study resonances and localized states in the vicinity of a one-dimensional (1D) barrier for the model where the Dirac Hamiltonian is complemented with the mass gap. To this goal we apply an analog of the WKB or semiclassical analysis to the case of the matrix Dirac equations. Using the expansion of the solution in power of $\hbar$ we generate a set of the so-called transport equations, which can be solved explicitly. Analysis of the scattering problem for a single peaked barrier is done in the leading order contribution. Explicit expression for the transmission and reflection coefficients are obtained by matching the WKB solution with the known uniform asymptotics of the so-called comparison (or reference) equation [33,34]. An universal Bohr-Sommerfeld-like condition is derived for both virtual and localized states and its influence on the density of single particle states is discussed. We shall consider the situation where the transitions between the two cones of the graphene spectrum can be neglected. The validity of this approximation follows from the fact that the potential is assumed adiabatic, so that its large momenta components that can induce the transition between the cones are negligible. In fact this adiabatic condition is also necessary for the validity of the WKB approximation itself.

\section{SEMICLASSICAL SOLUTION}

The Hamiltonian of a single layered graphene in the presence of electrostatic potential and modified by the gap is [2]

$$
H=v_{F}(\boldsymbol{\sigma} \cdot \boldsymbol{p})+g \sigma_{z}+\sigma_{0} U(x),
$$

where $v_{F}$ denotes the Fermi velocity, $\sigma=\left(\check{\sigma}_{x}, \check{\sigma}_{y}\right)$, with $\check{\sigma}_{i}$ being Pauli matrices acting in the space of vector functions $\boldsymbol{\Psi}=(u, v)$ that correspond to sublattices $A$ and $B$, respectively, $\sigma_{0}$ is the unit matrix, and $\mathbf{p}=\left(p_{x}, p_{y}\right)$ is the momentum operator. The potential $U(x)$ represents the external barrier, varying only in the $x$ direction. The second term in this Hamiltonian, with the constant $g=m v_{F}^{2}$, describes the mass gap in the quasiparticle spectrum. We assume that $U(x)$ has a single peak with the maximum at $x=0$ and that it vanishes as $|x| \rightarrow \infty$. Obviously the problem can be reduced to $1 \mathrm{D}$ one by taking the wave function in the form $\exp \left(i p_{y} y\right) \Psi(x)$.

In the analysis it is convenient to use the following dimensionless quantities that are related to the barrier potential, $\tilde{x}=x / D, \tilde{E}=E / U_{0}, \tilde{p}_{y}=v_{F} p_{y} / U_{0}$, and $\tilde{\hbar}=\hbar v_{F} / U_{0} D$, where $D$ is the characteristic width of the potential [defined as the distance between the points at which $U(x)$ is half its maximal value] and $U_{0}$ is its maximal value. We shall omit tildes when it does not lead to ambiguities. Stationary states of the system are obtained by solving the following equation:

$$
\left(\begin{array}{cc}
U+g & -i \hbar \partial_{x}-i p_{y} \\
-i \hbar \partial_{x}+i p_{y} & U-g
\end{array}\right) \boldsymbol{\Psi}=E \boldsymbol{\Psi},
$$

with the appropriate boundary conditions, where $\boldsymbol{\Psi}$ denotes a vector function in the pseudospin space. Following a standard WKB approach [33] we seek a solution to this stationary problem $\boldsymbol{\Psi}$ in the form of a series expansion over powers of the Planck constant as

$$
\boldsymbol{\Psi}=e^{i \frac{S}{\hbar}} \sum_{j=0}^{\infty}(-i \hbar)^{j} \boldsymbol{\Psi}_{j} .
$$

Substituting Eq. (3) into Eq. (2) and matching the powers of $\hbar$ we obtain a recurrent sequence of equations for $\boldsymbol{\Psi}_{j}$ as

$$
\hat{L} \boldsymbol{\Psi}_{j}=\sigma_{x} \frac{d}{d x} \boldsymbol{\Psi}_{j-1},
$$

where

$$
\hat{L}=\left(\begin{array}{cc}
\xi+g & \partial_{x} S-i p_{y} \\
\partial_{x} S+i p_{y} & \xi-g
\end{array}\right), \quad \xi=U-E,
$$

and $\boldsymbol{\Psi}_{-1}=0$. In the mathematical WKB theory Eq. (4) is referred to as the transport equation, which should not be confused with transport phenomena in matter.

At the lowest order, $j=0$, Eq. (4) is a homogeneous linear equation

$$
\hat{L} \Psi_{0}=0,
$$

which is solvable if the condition $\operatorname{det}(\hat{L})=0$ is satisfied. Resolving it we find the analog of the classical action $S$ in the form

$$
\begin{aligned}
S\left(x, x_{0}\right) & =\int_{x_{0}}^{x} p_{x} d x, \\
p_{x} & =\sqrt{\xi^{2}-P^{2}}, \quad P=\sqrt{g^{2}+p_{y}^{2}},
\end{aligned}
$$

where the sign of $p_{x}$ is defined by the branch of the root. Analogously to the WKB analysis in quantum mechanics, here the "classically allowed" domain is defined by the condition $\xi^{2}-P^{2}>0$, whereas $\xi^{2}-P^{2}<0$ defines "classically inaccessible" or forbidden domain and the turning points are defined by $\xi^{2}-P^{2}=0$.

Substituting Eq. (7) into Eq. (4) we find the leading order contribution in the form

$$
\boldsymbol{\Psi}_{0}=\boldsymbol{e} \psi_{0},
$$

where $\mathbf{e}$ is the corresponding eigenvector of the matrix equation (6) and the spatial dependence $\psi_{0}$ is arbitrary at this stage. The explicit form of the eigenvector reads as

$$
\mathbf{e}=\left(\begin{array}{c}
1 \\
\Theta
\end{array}\right), \quad \Theta=-\frac{p_{x}+i p_{y}}{\xi-g} .
$$

The next order equation

$$
\hat{L} \boldsymbol{\Psi}_{1}=\sigma_{x} \frac{d}{d x} \boldsymbol{\Psi}_{0}
$$

contains both the leading order $\boldsymbol{\Psi}_{0}$ and the next order $\boldsymbol{\Psi}_{1}$ contributions. The latter can be written in the form

$$
\boldsymbol{\Psi}_{1}=\mathbf{e} \psi_{1}+\mathbf{d} \xi_{1},
$$


where vector $\mathbf{d}$ is orthogonal to $\mathbf{e}$ and is explicitly written as

$$
\mathbf{d}=\left(\begin{array}{l}
1 \\
\Xi
\end{array}\right), \quad \Xi=\frac{p_{x}+i p_{y}}{\xi+g} .
$$

Substituting solution (11) into Eq. (10) and projecting the resulting equation onto $\mathbf{e}$, while using that $\mathbf{e}$ is the eigenvector of $\hat{L}$ with zero eigenvalue, we obtain the equation for $\psi_{0}$ as

$$
(\Theta+\bar{\Theta}) \frac{d \psi_{0}}{d x}+\frac{d \Theta}{d x} \psi_{0}=0,
$$

where $\bar{\Theta}$ is obtained from $\Theta$ in Eq. (9) by substituting $p_{y} \rightarrow-p_{y}$. For the classically allowed domain this yields the complex conjugate $\bar{\Theta}=\Theta^{*}$. The solution to Eq. (13) is easily found as

$$
\psi_{0}=\frac{1}{\sqrt{p_{x}}}\left[p_{x}-i p_{y}+i \frac{g}{p_{y}}(\xi-g)\right]^{1 / 2} .
$$

In the classically forbidden domain $p_{x} \rightarrow i p_{x}$ with $p_{x}=$ $\sqrt{P^{2}-\xi^{2}}$. A similar procedure of projecting the equation is applied at all orders of the expansion to obtain all terms in the expansion (3) as shown in Appendix A.

Expression for $\psi_{0}$ in Eq. (14) diverges at $p_{x}=0$. Similarly to the standard quantum mechanics, this divergence limits the applicability of the WKB expansion which is not valid close to turning points. Apart from this restriction the validity domain of the approach is defined by the condition that the next order contribution $\psi_{1}$ is small as compared with $\psi_{0}$. Interested readers can find details of the calculations of ratio $\psi_{1} / \psi_{0}$ in Appendix B. Analysis of the obtained expressions in various limiting cases leads to the following validity condition:

$$
\gamma\left|\frac{d \lambda_{x}}{d x}\right| \ll 1
$$

where $\lambda_{x} \propto p_{x}^{-1}$ is the quantum wavelength and $\gamma$ is a factor that depends on particular relations between parameters $p_{x}$, $p_{y}$, and $g$.

Apart from factor $\gamma$ the criterium in Eq. (15) coincides with that in standard quantum mechanics. In many cases $\gamma \ll 1$ and, therefore, the applicability of the WKB method for the Dirac equation is enhanced as compared with the Schrödinger equation. However, if the $g \gtrsim p_{x, y}$ this factor rises, $\gamma \gtrsim 1$. Still in this case the amplitude of $\psi_{0}$ in Eq. (14) also rises, which is a consequence that the "proximity" interval close to a turning point, where the WKB expansion fails, also increases. Thus, the WKB approximation for the Dirac equation works generally better than in the quantum mechanics far from the turning points. But the interval around the turning points where the method is not applicable becomes large when the gap increases.

\section{CLASSICAL ACTION AND STATE TYPES}

Stationary states in the system can be classified by comparing shifts in the energy of the bands induced by the barrier. The WKB approach introduces the analog of the classical action $S$ and momentum $p_{x}$ which are real in the classically allowed intervals and become complex in classically forbidden ones. Turning points, which separate allowed and forbidden intervals, are found from the condition $p_{x}=0$, which yields
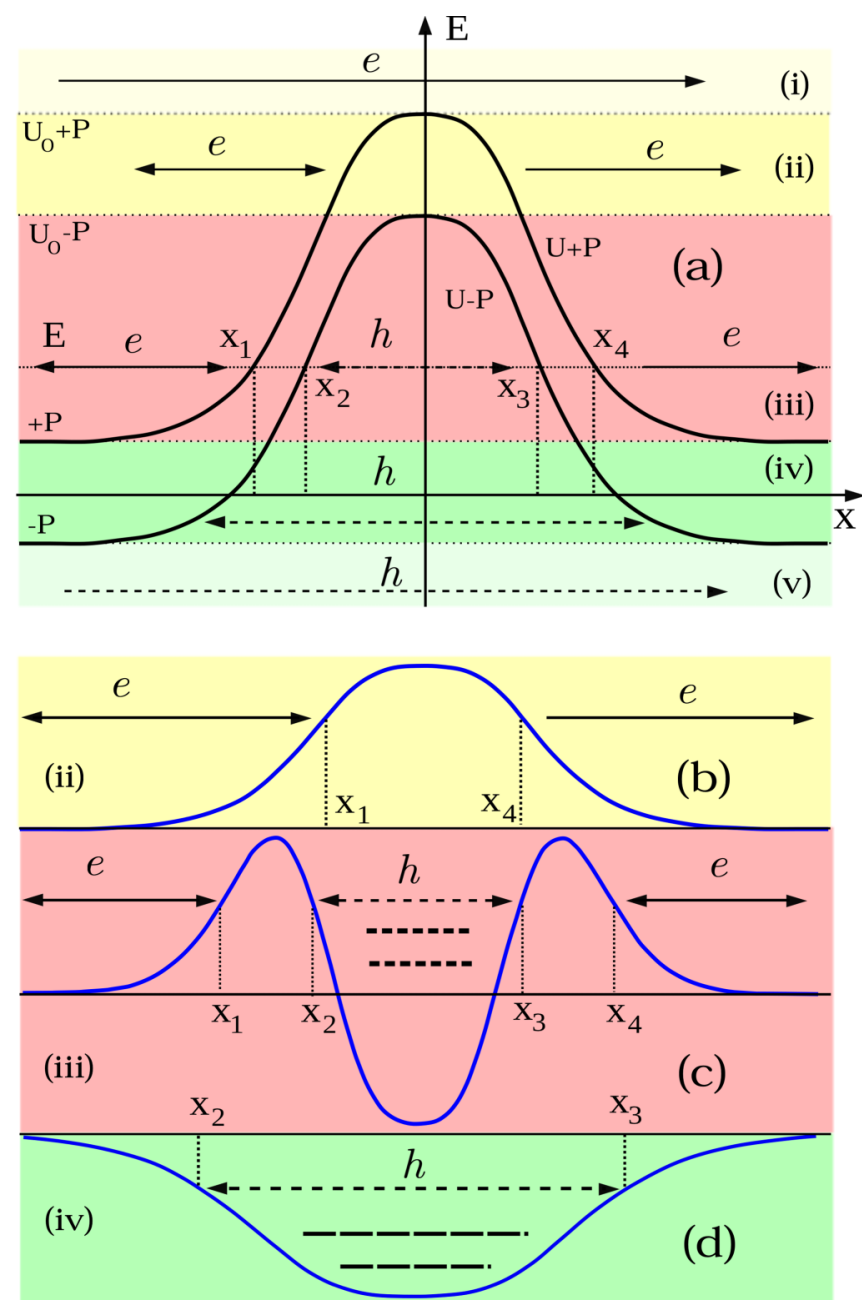

FIG. 1. (Color online) Different scattering states in intervals (i)(v), shaded by respective colors, for a single peaked barrier $U(x)$. Continuous black lines in (a) represent the shifted potential $U(x) \pm$ $P, x_{i}$ are turning points found by solving $E=U(x) \pm P$. (b)-(d) Illustrate scattering in energy intervals (ii)-(iv) [shaded by the same colors as in (a)]: (b) Interval (ii) with $e$-e tunneling of electrons, (c) interval (iii) with the resonant $e-h-e$ tunneling, and (d) interval (iv) with localized $h$ states. $e / h$ denote electrons/holes, blue lines give the effective potential $E^{2}-[U(x)-E]^{2}$, short dashed lines in (c) illustrate positions of virtual hole states, and long dashed lines in (d) represent localized hole states.

the equation

$$
U(x)=E \pm P,
$$

where $P>0$. Solving this equation is graphically illustrated in Fig. 1. For the potential of a chosen shape with a single extremum, this equation can have zero, two, or four different (real) solutions, labeled as $x_{i}, i=1, \ldots, 4$. We note that unlike quantum mechanics the role of the effective potential is played by the quantity $E^{2}-[U(x)-E]^{2}$, which is illustrated by blue lines in Figs. 1(b)-1(d).

We can distinguish five energy intervals (i)-(v) (see Fig. 1) with different types of scattering. For $U_{0}+P<E$ (i) there are no turning points, the entire space is classically allowed and there is standard over-the-barrier electron scattering. For 
$U_{0}-P<E<U_{0}+P$ (ii) the $\left[x_{1}, x_{4}\right]$ interval is classically forbidden. Figure 1(b) illustrates the corresponding effective potential through which electrons tunnel. In intervals (i) and (ii) scattering is thus similar to nonrelativistic quantum mechanics.

At lower energies nonconventional scattering emerges. In the interval $P<E<U_{0}-P$ (iii) two additional turning points are encountered, $x_{2}$ and $x_{3}$, leading to two classically forbidden intervals, $\left[x_{1}, x_{2}\right]$ and $\left[x_{3}, x_{4}\right]$. The classically allowed domain is subdivided into two intervals of electron states, $\left(-\infty, x_{1}\right]$ and $\left[x_{4}, \infty\right)$, and one of hole states, $\left[x_{2}, x_{3}\right]$. For this case scattering is schematically illustrated in Fig. 1(c), which shows the double barrier structure for the effective potential. An electron, coming from left, first tunnels through the barrier interval $\left[x_{1}, x_{2}\right]$ becoming a hole in the process, then travels freely in $\left[x_{2}, x_{3}\right]$ and then tunnels through the second barrier $\left[x_{3}, x_{4}\right]$, becoming an electron again. The conversion between the electron and hole states while passing through the barrier is qualitatively analogous to the Landau-Zener tunneling in semiconductors. The scattering amplitude has resonances due to the quasistationary or virtual hole states in $\left[x_{2}, x_{3}\right]$, schematically illustrated by dashed lines in Fig. 1(c). One notes that interval (iii) appears only when the hole and electron bands intersect due to the barrier potential, i.e., when $2 P<U_{0}$.

When the energy is lowered further reaching the interval $-P<E<P$ (iv), turning points $x_{1}$ and $x_{4}$ disappear at $\mp \infty$, leaving a single classically allowed interval $\left[x_{2}, x_{3}\right]$ as illustrated in Fig. 1(d). In this interval the hole states are localized in the transverse direction $x$ (the motion in the $y$ direction is free) that are referred to as the localized states for clarity. Finally, in interval $E<-P$ (v) the remaining turning points $x_{2}$ and $x_{3}$ disappear at infinity, and the entire space becomes classically allowed. In this interval holes scatter on the potential well.

The structure of the intervals (i)-(v) as function of the momentum component $p_{y}$ is illustrated in Fig. 2. Figures 2(a) and 2(b) illustrate, respectively, the cases where the potential

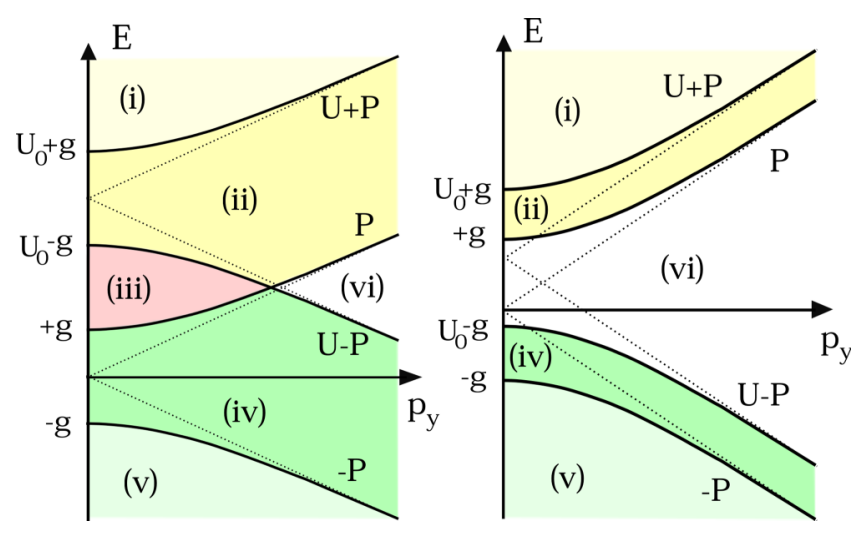

FIG. 2. (Color online) Band structure: Dependence of scattering intervals (i)-(v) (shaded respectively as in Fig. 1) on transverse momentum component $p_{y}$. Straight lines represent graphene with zero gap. (a) Illustrates the case $U_{0}>2 g$ with the resonant scattering in interval (iii), and (b) shows the system without such an interval. Localized states interval (iv) is present in both panels. Domain (vi) denotes a gap. does and does not lead to the intersection of the electron and hole bands in the energy scale. In all cases the boundaries of the electron band increases while that of the hole band decreases for larger $p_{y}$, as follows from Eq. (16). At $p_{y}>\sqrt{U_{0}^{2} / 4-g^{2}}$ the interval (iii) of the double barrier tunneling disappears. For a small barrier and/or large gap, $U_{0}<2 g$, the double barrier structure is not found for any $p_{y}$, as illustrated in Fig. 2(b). However, interval (iv) of the localized hole states exists for any $p_{y}$ irrespective of the potential depth, with the remark that the quantization conditions defines a minimal value of $U_{0}$, at which localized states become possible. For comparison the $p_{y}$ dependencies of the band boundaries in the gapless case $g=0$ are shown by dotted lines in Fig. 2. One notes an important difference: For the gapless case the resonant scattering interval (iii) exists for a barrier of arbitrary height.

\section{TUNNELING PROBABILITY}

Following standard scattering theory the scattering coefficients are defined using solutions with free particle asymptotes at infinity, which read as

$$
\boldsymbol{\Psi}(x)= \begin{cases}e^{i p_{x} x} \mathbf{e}_{1} a_{1}+e^{-i p_{x} x} \mathbf{e}_{2} a_{2}, & x \rightarrow-\infty, \\ e^{i p_{x} x} \mathbf{e}_{1} b_{1}+e^{-i p_{x} x} \mathbf{e}_{2} b_{2}, & x \rightarrow+\infty,\end{cases}
$$

where $\mathbf{e}_{1,2}$ are defined as in Eq. (9) with

$$
\Theta_{1,2}=-\frac{ \pm p_{x}+i p_{y}}{\xi-g}, \quad p_{x}=\sqrt{E^{2}-p_{y}^{2}-g^{2}} .
$$

Transmission and reflection coefficients are defined via the coefficients of the transfer matrix that connects coefficients $a_{1,2}$ and $b_{1,2}$ as

$$
\left(\begin{array}{l}
b_{2} \\
b_{1}
\end{array}\right)=\left(\begin{array}{ll}
T_{22} & T_{21} \\
T_{12} & T_{11}
\end{array}\right)\left(\begin{array}{l}
a_{2} \\
a_{1}
\end{array}\right) .
$$

The coefficients satisfy the relation $\left|a_{1}\right|^{2}-\left|a_{2}\right|^{2}=\left|b_{1}\right|^{2}-$ $\left|b_{2}\right|^{2}$, which follows from the conservation of the probability current $j \propto u^{*} v+v^{*} u$. The current conservation is also reflected in the coefficients of the transfer matrix $T$ which satisfy

$$
T_{22}=T_{11}^{*}, T_{21}=T_{12}^{*}, \quad \operatorname{det} T=1 .
$$

One can also introduce the unitary scattering matrix as

$$
\left(\begin{array}{l}
a_{2} \\
b_{1}
\end{array}\right)=\hat{S}\left(\begin{array}{l}
a_{1} \\
b_{2}
\end{array}\right), \quad \hat{S}=\left(\begin{array}{ll}
r_{1} & t \\
t & r_{2}
\end{array}\right),
$$

where

$$
t=\frac{1}{T_{22}}, \quad r_{1}=-\frac{T_{21}}{T_{22}}, \quad r_{2}=\frac{T_{12}}{T_{22}} .
$$

Reflection and transmission coefficients here satisfy standard relations $|t|^{2}+\left|r_{1,2}\right|^{2}=1$.

The coefficients of the transfer matrix are routinely obtained by matching the semiclassical solution with the uniform asymptotic solutions, obtained using the asymptotic form of the potential in the vicinity of the turning points, where the effective potential $\sqrt{[U(x)-E]^{2}-P^{2}}$ can be approximated by the linear function, for which exact solution is given by Airy functions. Using known asymptotic expansions of Airy functions at $x \rightarrow \pm \infty$ we match them with the WKB solutions 
for the linear potential, thereby obtaining the scattering coefficients on both sides of the turning points. This matching procedure has to be repeated at all four possible turning points. The validity of this approach relies on the assumption that the separation between the turning points is sufficiently large so that the WKB approximation can be used in the intervals between them.

This assumption, however, fails when the turning points become too close one to another. In particular, this is the case for normal scattering $p_{y}=0$ and zero mass gap, where $x_{1,3}$ merge with $x_{2,4}$, respectively. This limiting case is encountered in the analysis of the resonant scattering in energy interval (iii). In order to obtain the solution that covers this limit as well, we may approximate the effective barrier using the harmonic potential in both intervals $\left[x_{1,3}, x_{2,4}\right]$, for which the exact solution is also known. In this work this is done using the elegant formalism of the comparison equation [34]. The advantage of this formalism is its accuracy in describing both limiting cases of the small and large intervals between pairs of the turning points $x_{1,2}$ and $x_{3,4}$ and thus the crossover between the regimes of Klein and Landau-Zenner tunneling. Details of the derivation of the comparison equation and the outline of the procedure of matching its solutions with the WKB expansion is outlined in Appendix C. We note in passing that this formalism also becomes inadequate in the special case when all four turning points are close. This situation appears difficult as the potential in this case should be approximated by the fourth order polynomial, an exact solution for which is not known. However, this case is encountered only for the normal scattering and under conditions that energy of a particle is close to $U_{0}$ and that the gap is small.

After relatively straightforward but cumbersome calculations, some necessary details of which are found in Appendix $\mathrm{C}$, we obtain the following coefficients of the transfer matrix:

$$
\begin{aligned}
& T_{11}=e^{Q_{1}+Q_{2}}\left(s_{1} s_{2} e^{i\left(\theta_{1}+\theta_{2}+K\right)}+e^{-i K}\right), \\
& T_{12}=e^{-i \alpha+Q_{1}+Q_{2}}\left[s_{2} e^{i\left(\theta_{2}+K\right)}+s_{1} e^{-i\left(\theta_{1}+K\right)}\right] .
\end{aligned}
$$

In these expressions

$$
s_{i}=\sqrt{1-\exp \left(-2 Q_{i}\right)},
$$

$Q_{1,2}$ and $K$ are the "classical" action between turning points

$$
\begin{aligned}
Q_{1} & =\frac{1}{\hbar}\left|S\left(x_{2}, x_{1}\right)\right|, \quad Q_{2}=\frac{1}{\hbar}\left|S\left(x_{4}, x_{3}\right)\right|, \\
K & =\frac{1}{\hbar}\left|S\left(x_{3}, x_{2}\right)\right|,
\end{aligned}
$$

the phases are

$$
\begin{aligned}
& \alpha=\frac{\pi}{2}-\theta+\arg \left[\frac{i p_{y}+g+p_{x}^{-}+E}{i p_{y}+g-p_{x}^{-}+E} \frac{E-p_{x}^{-}}{P}\right], \\
& \theta_{j}=\frac{Q_{j}}{\pi}\left(1-\log \left[\frac{Q_{j}}{\pi}\right]\right)-\arg \Gamma\left[1-i \frac{Q_{j}}{\pi}\right]-\frac{\pi}{4},
\end{aligned}
$$

with $\Gamma(x)$ being the $\Gamma$ function and

$$
\theta=\arg \left(p_{x}^{-}+i p_{y}\right), \quad p_{x}^{-}=\sqrt{E^{2}-P^{2}} .
$$

It is easy to see that in the limit $g=0$ the coefficients in Eqs. (23) coincide with the known results for the gapless case [29]. Using relations (22) one can calculate the reflection and transmission coefficients. The final expressions simplify considerably for a symmetric barrier, for which $Q_{2}=Q_{1}=Q$ and $\theta_{2}=\theta_{1}=\theta$ and the scattering coefficients become

$$
\begin{aligned}
t & =e^{i \theta}\left[\cos (P+\theta)\left(2 e^{2 Q}-1\right)+i \sin (P+\theta)\right]^{-1}, \\
r & =2 t e^{2 Q+i \alpha} \sqrt{1-e^{-2 Q}} \cos (P+\theta) .
\end{aligned}
$$

Oscillations in the coefficients is a manifestation of resonant tunneling: The transmission sharply increases when the energy of a scattered particle matches that of a virtual hole state. We note that with the exception of the phase factor $\alpha$, Eq. (28) coincides with the corresponding expressions in the gapless case when $p_{y}$ is substituted by $P$. This coincidence (without the phase factor) confirms an intuitive expectation which follows from the structure of the eigenvalue problem.

\section{RESONANCES AND LOCALIZED STATES}

Transmission through the barrier in the resonant energy interval (iii) is illustrated in Fig. 3, where the tunneling coefficient is calculated for the potential $U(x)=\operatorname{sech}(x)$ as a function of momentum components $p_{x}$ and $p_{y}$ of a scattered particle. The resonances, where $|t| \approx 1$, are clearly seen in the figure as narrow stripes. Contrary to the gapless case, the regime of complete Klein tunneling, with $|t|=1$ at $p_{y}=0$, is absent here. This follows directly from Eq. (28), where in the gapless case the turning points merge at $p_{y}=0\left(x_{1}=x_{2}\right.$ $\left.x_{3}=x_{4}\right)$ yielding $Q=0$ and thus $t=\exp (i P)$. However, when $g \neq 0$, the turning points remain separate at all values of $p_{y}$ and, therefore, $Q \neq 0$. Full transmission can still be achieved but only at a resonance condition

$$
K(E)+\theta=\pi\left(n+\frac{1}{2}\right),
$$

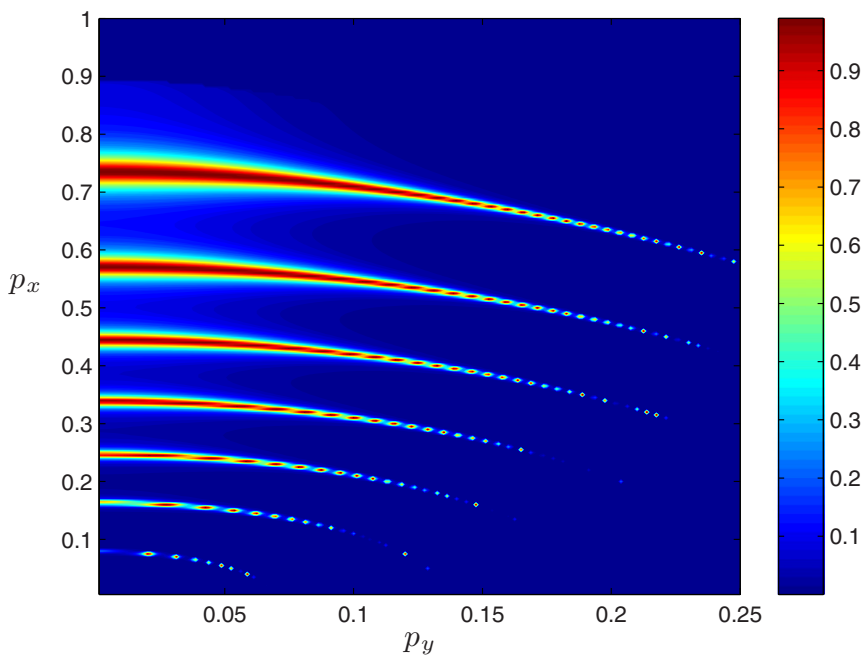

FIG. 3. (Color online) Bar diagram for the absolute value of the transmission coefficient $|t|^{2}$ as a function of momentum components $p_{x}$ and $p_{y}$, calculated from the WKB expression (28) for barrier $U=$ $\operatorname{sech}(x)$ for $g=0.1$. The stripes (red) are the domains of resonant scattering $|t| \approx 1$ that correspond to positions of the virtual states given by Eq. (29) illustrated in Fig. 1. 
where the $E$ dependence of $K$ is defined by Eq. (23) and $n=0,1, \ldots, N_{\max }$ is an integer denoting resonances. For any finite potential this equation has a finite number of roots that defines the total number $N_{\max }$ of the stripes with maximal transmission. Equation (29) appears as a standard Bohr-Sommerfeld quantization condition.

The width of the resonances (or lifetime of the virtual levels) is found by solving the eigenvalue problem with the boundary conditions corresponding to localized states. This yields the equation $T_{22}(E)=0$ which with the help of Eq. (23) reads explicitly as

$$
K(E)+\theta+\frac{i}{2} \log \left(1-e^{-2 Q}\right)=\pi\left(n+\frac{1}{2}\right) .
$$

Contrary to Eq. (29), it contains an additional complex contribution and, therefore, its roots are also complex reflecting the virtual character of the localized states. We search for the roots by explicitly separating its real and complex parts, $\varepsilon=E+i \Gamma$. Assuming that the tunneling rate is small, $\exp (-Q) \ll 1$, we calculate $\Gamma$ perturbatively. In the largest order Eq. (30) reduces to Eq. (29), and therefore yields transmission resonances. $\Gamma$ is obtained at the next order as

$$
\Gamma=\frac{\hbar w}{2 \Delta t}, \quad w=-\log \left(1-e^{-2 Q}\right), \quad \Delta t=\frac{d K(E)}{d E} .
$$

This expression allows for a physically transparent interpretation if one recalls that $w$ in this limit yields the tunneling probability and $\Delta t$ can be considered as the time a particle spends in the barrier. Then Eq. (31) coincides with a standard WKB estimation for the width in quantum mechanics [28].

Due to the hole-electron transmutation in the scattering the dependence of the solution of Eq. (29) on the quantum number $n$ is inverse: $E$ decreases at larger $n$. From Eq. (31) one can see that $\Gamma$ is a decreasing function on $E$; this also follows from the fact that the distance between turning points $x_{1}$ and $x_{2}$ increases at smaller $E$ (cf. Fig. 1). This implies that $\Gamma$ decreases at larger $p_{y}$. Thus the transmission peaks are generally sharper at smaller energy and also at smaller $p_{x}$ and $p_{y}$, which is seen in Fig. 3. When the energy decreases further and reaches the boundary $E=P$ between domain (iii) of the resonant scattering and domain (iv) of localized hole states, $\Gamma$ vanishes.

The existence of hole states localized in the vicinity of the barrier is often overlooked [29], despite the fact that such states are found in a much wider parameter interval than the resonant scattering. Domain (iv) of the localized states exists for arbitrary $U_{0}$ and appears in both panels in Fig. 2, while domain (iii) of the resonance scattering is found only in Fig. 2(a) where the condition $U_{0} \geqslant 2 g$ is fulfilled. Also, localized states are found at energies $E \leqslant U_{0}-P$ when the longitudinal momentum $p_{y}$ lies in the interval $\sqrt{E^{2}-g^{2}} \leqslant$ $p_{y} \leqslant \sqrt{\left(E-U_{0}\right)^{2}-g^{2}}$.

Eigenvalues of the localized states are also obtained from the WKB solution by imposing the decaying boundary conditions on the solutions. The calculations are similar to the case of resonant scattering and yield Eq. (30) for the eigenvalues, in which, however, the limit $Q \rightarrow \infty$ must be taken. The eigenstates are then defined by Eq. (29) with $\theta=0$ where quantum number $n$ starts from $N_{\max }+1$. The quantization introduces limitations on the existence and the total number of the localized states. One can estimate the total number of bound and quasibound states in the system as

$$
N_{\text {bound }} \approx \frac{2}{\pi} K(-P)=\frac{1}{\pi \hbar}\left\{2 \int_{-\infty}^{\infty} d x \sqrt{U(U+2 P)}-1\right\},
$$

where we extended the integration limits $x_{2,3}$ to infinity. Thus the number of localized states increases at larger $P$. Even if no such states are possible initially at $p_{y}=0$ or $P=g$, they will appear at large $p_{y}$, where the number of such states is estimated as

$$
N_{\text {bound }} \approx \beta \sqrt{P}, \quad \beta=\frac{\sqrt{2}}{\pi \hbar} \int_{-\infty}^{\infty} d x \sqrt{U} .
$$

This expression marks a qualitative difference with standard quantum mechanics where the number of transverse bound states in a $1 \mathrm{D}$ well does not depend on the value of the $p_{y}$ component. Here it rises with both $p_{y}$ and the gap $g$.

Localized states modify the single particle density of states (DOS) by adding additional divergent contribution. In the absence of the barrier the DOS in gapless graphene is $\rho(E) \propto$ $E$, which approaches zero at the Dirac point $E=0$. When the gap opens, $g \neq 0$, the DOS becomes $\rho(E) \propto \sqrt{E^{2}-g^{2}}$, which goes to zero at the gap edges $|E| \rightarrow g$. When the barrier potential is present the DOS comprises additional contributions due to the subbands formed by the localized states. In the limit of small $p_{y}$ and thus the highest energy of the hole subband $n$, one obtains an additional contribution to the DOS as $\rho_{n}(E) \propto p_{y}^{-1}$, as the localized states here are still extended in the $y$ direction, resulting in effective 1D states. This contribution diverges in the limit $p_{y} \rightarrow 0$ yielding a van Hove singularity at $E=E_{n}\left(p_{y}=0\right)$. There is an important difference with the gapless case $g=0$, where the singularity is absent because the system enters the regime of full Klein tunneling $\left(|t|=1\right.$ at $\left.p_{y}=0\right)$ and both localized and virtual states are absent. Thus, the gapless system does not reveal singular contributions in the DOS due to the barrier. We can also distinguish cases $g<U_{0}$ and $g>U_{0}$, depicted in Figs. 1(a) and 1(b), respectively. In the former the singularities are found inside the gap $-g<E<g$, while at $U_{0}-g<E<g$ the virtual states can lead to appearance of peaks in the DOS, if the resonances do not overlap. In the latter case singularities can appear when $-g<E<U_{0}-g$.

\section{CONCLUSIONS}

In summary, we have presented a semiclassical analysis of scattering on a smooth 1D potential barrier in single layered graphene with a gap. The derivation of the WKB approach follows a standard strategy of expanding the vector wave function in powers of the Planck constant which allows us to explicitly obtain the contributions at each order by solving the corresponding transport equations. We note that a similar approach can also be developed for more complicated systems such as multilayered graphene. Analysis of the scattering on the barrier potential is done within the leading order of the WKB expansion. Using the analog of the classical action several qualitatively different stationary states have been identified, including those of resonant scattering and hole states localized in the vicinity of the barrier. WKB 
expressions were obtained for the wave functions, transmission coefficients, energies of the resonances and localized states, as well as for the resonance widths. Apart from the phase factor the expressions obtained in this work have a functional form similar to that derived earlier for the gapless case. However, unlike the latter, the system with nonzero gaps exhibits reflectionless Klein tunneling only at resonances even for the scattering at normal angle. Positions of the virtual and the localized states are defined by the same Bohr-Sommerfeld-like quantization condition. Unlike standard quantum mechanics, here the number of localized states increases at larger values of the longitudinal momentum component and at larger gap values, so that such states are always present in the system. It was also demonstrated that resonances and localized states can modify the single particle DOS by introducing van Hove singularities. Such states may be important in the electrical transport in the system. However, discussion of this aspect is beyond the scope of this work.

\section{ACKNOWLEDGMENT}

M.D.C. acknowledges the Belgian Science Policy (BELSPO Back to Belgium Grant).

\section{APPENDIX A: SOLUTION OF THE TRANSPORT EQUATIONS}

Equation (4) can be solved in the general form by choosing the solution in the form

$$
\boldsymbol{\Psi}_{j}=\mathbf{e} \psi_{j}+\mathbf{d} \chi_{j} .
$$

By substituting this into Eq. (4) one obtains

$$
\hat{L} \mathbf{d} \chi_{j}=\sigma_{x}\left(\mathbf{e} \frac{d \psi_{j-1}}{d x}+\mathbf{d} \frac{d \chi_{j-1}}{d x}\right),
$$

where we used the fact that $\mathbf{e}$ is the eigenvalue, $\hat{L} \mathbf{e}=0$. Multiplying this equation by $\overline{\mathbf{e}}$ and $\overline{\mathbf{d}}$ from the left-hand side and evaluating the resulting scalar products we obtain two equations

$$
\begin{aligned}
& (\Theta+\bar{\Theta}) \frac{d \psi_{j-1}}{d x}+\frac{d \Theta}{d x} \psi_{j-1}+(\Xi+\bar{\Theta}) \frac{d \chi_{j-1}}{d x} \\
& +\frac{d \Xi}{d x} \chi_{j-1}=0, \\
& (\Theta+\bar{\Xi}) \frac{d \psi_{j-1}}{d x}+\frac{d \Theta}{d x} \psi_{j-1}+(\Xi+\bar{\Xi}) \frac{d \chi_{j-1}}{d x} \\
& +\frac{d \Xi}{d x} \chi_{j-1}-4 \xi \frac{\xi-g}{\xi+g} \chi_{j}=0 .
\end{aligned}
$$

The first equation yields $\psi_{j-1}$ from $\chi_{j-1}$ in the explicit form

$$
\begin{aligned}
\psi_{j-1}= & \psi_{j-1}^{(0)} e^{F(x)}-\int_{x_{0}}^{x} d y e^{F(x)-F(y)} \\
& \times\left\{(\Xi+\bar{\Theta}) \frac{d \chi_{j-1}}{d x}+\frac{d \Xi}{d x} \chi_{j-1}\right\},
\end{aligned}
$$

where $\psi_{j-1}^{(0)}=\psi_{j-1}\left(x_{0}\right)$ is the boundary condition and

$$
F(x)=-\int_{x_{0}}^{x} \frac{\Theta^{\prime}}{\bar{\Theta}+\Theta} d x .
$$

Using Eq. (A2) one obtains $\chi_{j}$ in the form

$$
\chi_{j}=\frac{\bar{\Xi}-\bar{\Theta}}{4 \xi} \frac{\xi+g}{\xi-g}\left(\frac{d \phi_{j-1}}{d x}+\frac{d \chi_{j-1}}{d x}\right) .
$$

Equations (A5)-(A7) yield explicit solutions for all coefficients in the WKB expansion for the states. For scattering problems the boundary conditions for $\psi_{j}$ and $\chi_{j}$ are typically defined by the asymptotic solutions. In particular, when the potential vanishes at infinity one obtains that $\psi_{0}$ is constant while all other $\psi_{j}$ and $\chi_{j}$ are zero.

\section{APPENDIX B: VALIDITY OF WKB EXPANSION}

The general solution of Eq. (13) is

$$
\psi_{0}=A \exp \left(-\int \frac{1}{\Theta+\bar{\Theta}} \frac{d \Theta}{d x} d x\right),
$$

with $A$ being constant. For the leading correction $\psi_{1}$ we solve Eqs. (A3) and (A4) with $j=1$. Equation (A4) yields

$$
\chi_{1}=f \phi_{0}, \quad f=\frac{1}{4 \xi} \frac{\xi+g}{\xi-g} \frac{\bar{\Theta}-\bar{\Xi}}{\Theta+\bar{\Theta}} \frac{d \Theta}{d x} .
$$

Equation (A3) is solved using the substitution $\phi_{1}=F \phi_{0}$ which helps to transform it into the following form:

$$
(\Theta+\bar{\Theta})\left(\frac{d F}{d x}+\frac{d f}{d x}\right) \phi_{0}+\frac{d}{d x}\left\{(\Xi-\Theta) f \phi_{0}\right\}=0,
$$

which with the help of Eq. (13) is further recast into

$$
\frac{d F}{d x}+\frac{d}{d x}\left(\frac{\bar{\Theta}+\Xi}{\Theta+\bar{\Theta}} f\right)=\frac{1}{\Theta+\bar{\Theta}} \frac{d \bar{\Theta}}{d x} f .
$$

Solution to this equation reads as

$$
\begin{aligned}
F= & -\frac{1}{4 \xi} \frac{\xi+g}{\xi-g} \frac{\bar{\Theta}+\Xi \bar{\Theta}-\bar{\Xi} \frac{d \Theta}{\Theta+\bar{\Theta}} \frac{\bar{\Theta}+\bar{\Theta}}{d x}}{} \\
& +\int \frac{1}{4 \xi} \frac{\xi+g}{\xi-g} \frac{\bar{\Theta}-\bar{\Xi}}{(\Theta+\bar{\Theta})^{2}} \frac{d \Theta}{d x} \frac{d \bar{\Theta}}{d x} d x .
\end{aligned}
$$

The condition of the applicability of the WKB approach away from turning points is that ratio $\psi_{1} / \psi_{0}$ is small. This yields

$$
|\hbar F| \ll 1 \text {. }
$$

This condition can be written in a simplified form in the important cases of particular relations between $p_{x}, p_{y}$, and $g$.

We consider first the case of large normal momentum $p_{x} \gg$ $\left\{p_{y}, g\right\}$. In this case the following leading contributions to $F$ can be extracted:

$$
F=-\frac{1}{4} \frac{P^{2}}{p_{x}^{2}} \frac{p_{x}^{\prime}}{p_{x}^{2}}-\frac{1}{8} \int \frac{P^{2}}{p_{x}^{2}} \frac{p_{x}^{2}}{p_{x}^{3}} d x,
$$

$p_{x}^{\prime}=d p_{x} / d x$. Both contributions in this expressions are of the same order, which can be seen, e.g., by evaluating the integral in the right-hand side by parts. Using the first term in this result and writing the derivative of $p_{x}$ via the derivative of the wavelength $\lambda_{x}\left(\hbar p_{x}^{\prime} / p_{x}^{2} \propto \lambda_{x}^{\prime}\right)$, the applicability condition for 
the $\mathrm{WKB}$ expansion is recast as

$$
\frac{P^{2}}{p_{x}^{2}}\left|\frac{d \lambda}{d x}\right| \ll 1
$$

In the opposite case $p_{x} \ll\left\{p_{y}, g\right\}$ one obtains

$$
F=-\frac{1}{4} \frac{P(P+g)}{p_{y}^{2}} \frac{p_{x}^{\prime}}{p_{x}^{2}}+\frac{i}{8} \frac{P+g}{P-g} \frac{1}{p_{y}} \int \frac{p_{x}^{\prime 2}}{p_{x}^{2}} d x .
$$

Taking the first term in this expression and rewriting the derivative as above we obtain the applicability condition in the form

$$
\frac{P(P+g)}{p_{y}^{2}}\left|\frac{d \lambda}{d x}\right| \ll 1 .
$$

The remaining possibilities are two intermediate cases: (a) $p_{y} \ll p_{x} \ll g$ and (b) $g \ll p_{x} \ll p_{y}$. In case (a) we obtain

$$
F=-\frac{g^{2}}{2} \frac{p_{x}^{\prime}}{p_{x}^{4}}-\frac{g^{2}}{2} \int \frac{p_{x}^{\prime 2}}{p_{x}^{5}} d x
$$

which yields the condition

$$
\frac{g^{2}}{p_{x}^{2}}\left|\frac{d \lambda}{d x}\right| \ll 1
$$

In case (b) $F$ can be approximated as

$$
F=-\frac{1}{4} \frac{p_{x}^{\prime}}{p_{x}^{2}}-\frac{i}{8} \frac{1}{p_{y}} \int \frac{p_{x}^{2}}{p_{x}^{2}} d x,
$$

leading to the condition

$$
\left|\frac{d \lambda}{d x}\right| \ll 1
$$

In the last case the applicability condition coincides with that in the quantum mechanics. In all other situations it contains additional factors, as follows from Eqs. (B8)-(B12). Equation (B8) is obtained for the case $\left\{p_{y}, g\right\} / p_{x} \ll 1$. Therefore, the validity domain of the WKB approach increases as compared with quantum mechanics given by Eq. (B14). Contrary to this case, in Eq. (B12) the additional factor can be much greater than unity, which follows from the condition $g \gg p_{x}$ used in its derivation. Finally, the value of the additional factor in Eq. (B10) varies from unity in the limit $p_{y} \gg g$ to large values, when $g \gg p_{y}$. One can thus see that if the gap $g$ is large the domain of applicability of the the WKB method for the Dirac equation has a tendency to decrease as compared to the Schrödinger equation. However, we note that in this limit the system is already in the vicinity of the turning point where the amplitude of the solution $\psi_{0}$ in Eq. (14) sharply rises.

We arrive to the conclusion, mentioned in the text, that when the system is away from the turning points the validity of the WKB expansion for the Dirac equation is generally enhanced in comparison with the Schrödinger equation. However, the interval in the vicinity of the turning points where the WKB approach is not applicable is larger than that in quantum mechanics and, furthermore, it rises when the gap increases.

\section{APPENDIX C: SOLUTION FOR TURNING POINTS: COMPARISON EQUATION METHOD}

Calculation of transfer matrix (19) requires finding relations between coefficients of the scattering solutions in Eq. (17). Within the WKB expansion this can be in principle done by calculating jumps in coefficients at the Stockes and antiStockes lines. In practice, however, a much more popular and simpler approach is to compare the WKB asymptotics with the more accurate solution in the vicinity of the turning points, where the WKB approximation fails. In conventional quantum mechanics such a solution is obtained by using a linear approximation for the potential close to the turning points. For the Dirac equation, as discussed in the text, this approximation fails in the limit of Klein tunneling for the normal angle scattering with small gaps. In this limit the effective potential, defined by $p_{x}$ in Eq. (7), is a function with a single maximum between two turning points for electron and hole states $x_{1}$ and $x_{2}$, respectively (or $x_{3}$ and $x_{4}$ ) [see Figs. 1(a) and $1(\mathrm{c})]$. Obviously the simplest solvable approximation for such potential in the interval $\left[x_{1}, x_{2}\right]$ is the second order polynomial, i.e., the harmonic potential. Using the Taylor expansion of the effective potential at the point of its maximum one obtains necessary parameters of this approximate potential and then proceeds to the standard routine of finding the exact solution and relating it to the WKB asymptotic. It is clear, however, that this approximation is suitable only when interval $\left[x_{1}, x_{2}\right]$ is relatively small. At larger intervals it needs be replaced by two separate linear approximations at $x_{1}$ and $x_{2}$, with two separate scattering coefficients matching problems to be solved for each of them. Therefore, obtaining a WKB solution, applicable in both limits of large and small $p_{x}$, for arbitrary shape of potential $U(x)$ is impossible within this approximation.

In this work we employ a different and more general approach referred to as the comparison equation method [34]. Unlike the Taylor expansion it relates the parameters of the approximate potential with the integral characteristics of the effective potential. At small intervals $\left[x_{1}, x_{2}\right]$ it reduces to the harmonic approximation described above, while at larger intervals it coincides with the results obtained by using the linear approximations at the turning points. Besides, it allows for a simple generalization to the complex potentials that is, as we shall see, the case of Dirac equation. Although popular in mathematical physics, this approach is not particularly well known in the community of physicist working with the WKB approximation. Here we provide a necessary introduction needed to understand the derivation of our results quoted in the text.

In order to simplify the analysis we first exclude one of the component functions in vector $\boldsymbol{\Psi}=(u, v)$ by first applying linear transformation

$$
W=\frac{1}{2}(u+v), \quad V=\frac{1}{2}(u-v),
$$

which helps to rewrite original system (2) in the form

$$
\begin{aligned}
& (U-E) W+\left(g+i p_{y}\right) V-i \hbar \frac{d W}{d x}=0, \\
& (U-E) V+\left(g-i p_{y}\right) W+i \hbar \frac{d V}{d x}=0 .
\end{aligned}
$$


Then we express $V$ from the first of these equations as

$$
V=\frac{1}{g+i p_{y}}\left\{i \hbar \frac{d W}{d x}+(E-U) W\right\},
$$

and exclude it from the second equation which becomes

$$
\hbar^{2} \frac{d^{2} W}{d x^{2}}+\mathcal{Q} W=0,
$$

where the effective potential is given by

$$
\mathcal{Q}=p_{x}^{2}+i \hbar \frac{d U}{d x},
$$

and $p_{x}=\sqrt{\xi^{2}-P^{2}}$ is defined by Eq. (7). Equation (C4) appears in the form of a standard Schrödinger equation (with zero eigenvalue), however, unlike standard quantum mechanics the effective potential $\mathcal{Q}$ in is now complex. We now rewrite this equation introducing a new variable $z$ by the following implicit transformation:

$$
\mathcal{Q}=\left(a^{2}-\frac{z^{2}}{4}\right)\left(\frac{d z}{d x}\right)^{2},
$$

where parameter $a$ (generally complex) will be defined later. Seeking a solution to Eq. (C4) in the form $W=\mathcal{W} / \sqrt{z^{\prime}}$ we obtain the following equation for $\tilde{W}$ regarded as a function of $z$ :

$$
\hbar^{2} \frac{d^{2} \mathcal{W}}{d z^{2}}+\left(a^{2}-\frac{z^{2}}{4}-\tilde{U}\right) \mathcal{W}=0
$$

where

$$
\tilde{U}=\frac{\hbar^{2}}{2\left(z^{\prime}\right)^{3 / 2}} \frac{d}{d x}\left(\frac{z^{\prime \prime}}{\left(z^{\prime}\right)^{3 / 2}}\right) .
$$

It can be demonstrated that potential $\tilde{U}$ is of the next order in powers of $\hbar$ will be neglected. In order to see this we search solution $\mathcal{W}$ as the function of scaled variable $\zeta=z / \sqrt{\hbar}$. Substituting this into Eq. (C7) and representing the transformation parameter as

$$
a^{2}=\hbar\left(v+\frac{1}{2}\right)
$$

one finds that all terms in Eq. (C7) are of the order $O(\hbar)$ except the potential $\tilde{U}$. The latter is of the order $O\left(\hbar^{3}\right)$ and therefore can be neglected in the WKB limit $\hbar \rightarrow 0$ leaving the following equation:

$$
\frac{d^{2} \mathcal{W}}{d \zeta^{2}}+\left(v+\frac{1}{2}-\frac{\zeta^{2}}{4}\right) \mathcal{W}=0
$$

It describes motion of a quantum particle in the quadratic potential and is customarily referred to as the comparison equation reflecting its purpose that is comparing its solution with the original Eq. (C4). One can now see that the choice of the scaling above and the consequent elimination of the last term in Eq. (C7) in fact follows from the harmonic approximation of the effective potential $\mathcal{Q}$. We also note that the imaginary part in $\mathcal{Q}$ in Eq. (C5) generates terms $\propto \hbar^{2}$ in Eq. (C7) that are still kept.

An arbitrary solution of Eq. (C10) are given by a linear combination of two parabolic cylinder functions $D_{v}(x)$ as

$$
\mathcal{W}=A D_{v}(\zeta)+B D_{-1-v}(i \zeta)
$$

with constant $A$ and $B$.
Solutions (C11) plays the same role as the Airy functions for the linear approximation for the potential in the vicinity of the turning point in quantum mechanics. The subsequent calculation of the transfer matrix $S$ in Eq. (19) essentially follows the standard routine described in textbooks on quantum mechanics. According to this procedure we compare the asymptotes of Eq. (C11) with the WKB expansion (17). However, since the calculations are done for the Dirac vector eigenvalue problem, one must return to the original functions $u$ and $v$ by finding $V$ from Eq. (C3) and then using the inverse of transformation $(\mathrm{C} 1)$.

Another nonstandard part of the matching procedure is that transformation (C6) must be inverted to return back to original coordinate $x$. We start the analysis of the transformation with noting that by virtue of Eq. (C6) two roots of equation $\mathcal{Q}=0$ ( $\tilde{x}_{1}$ and $\tilde{x}_{2}$ ) correspond to $z_{1,2}= \pm 2 a$. It is clear that $\tilde{x}_{1,2}$ do not coincide with the turning points $x_{1,2}$ due to nonzero imaginary contribution to $\mathcal{Q}$ in Eq. (C4). One also notes that the limits $z \rightarrow \pm \infty$ correspond to the limits $x \rightarrow \pm \infty$, so that the asymptotic expansions at large coordinates $x, z$ are needed.

We now complete the description of the transformation by finding its parameter $a$ from effective potential $\mathcal{Q}$ close to turning points $x_{1,2}$. To this aim we take the integral of both sides of Eq. (C6) which yields the equality

$$
\int_{\tilde{x}_{1}}^{\tilde{x}_{2}} \sqrt{\mathcal{Q}} d x=\int_{-2 a}^{2 a} \sqrt{a^{2}-\frac{z^{2}}{4}} d z .
$$

The integral of the right-hand side of this equation is evaluated exactly yielding $\pi a^{2}$. The integral on the left-hand side is expanded with respect to $\hbar$. The integration limits $\tilde{x}_{1,2}$ are obtained by expanding the corresponding equation $\mathcal{Q}=0$. To the leading order its roots are found as

$$
\tilde{x}_{2}-x_{2}=x_{1}-\tilde{x}_{1}=i \frac{\hbar}{P} .
$$

Substituting this and the expansion of $\mathcal{Q}$ into the integral on the left-hand side in Eq. (C12) we obtain two largest contributions as

$$
\int_{\tilde{x}_{1}}^{\tilde{x}_{2}} \sqrt{\mathcal{Q}} d x=i \hbar Q_{1}+\frac{\pi}{2} \hbar,
$$

where $Q_{1}$ is given by Eq. (25). Comparing the integrals on both sides we obtain

$$
a^{2}-\frac{\pi}{2}=i Q_{1}, \quad v=\frac{i}{\pi} Q_{1},
$$

so that the transformation parameters given by the "action" calculated under the barrier between the turning points. We note in passing that it is this integral relation between $a$ and the effective potential which makes the comparison equation method advantageous: It offers a good accuracy of the approach far beyond the limit of small distance between the turning points and thus describes the crossover between the Klein and the normal tunneling regimes by a single expression.

An explicit relation between $x$ and $z$ away from the turning points, needed for the asymptotes of solution (C11), is obtained 
similarly by using the integral form of Eq. (C6). At $x<x_{1}$ and $x>x_{2}$ this yields, respectively,

$$
\int_{x}^{\tilde{x}_{1}} \sqrt{\mathcal{Q}} d x=\int_{z}^{-2 a} \sqrt{a^{2}-\frac{z^{2}}{4}} d z
$$

and

$$
\int_{\tilde{x}_{2}}^{x} \sqrt{\mathcal{Q}} d x=\int_{2 a}^{z} \sqrt{a^{2}-\frac{z^{2}}{4}} d z .
$$

The integrals on the right-hand side are evaluated exactly and their asymptotes at large $|z| \gg|2 a|$ are obtained in both cases as

$$
i \frac{z^{2}}{4}-i a^{2} \ln |z|-i \frac{a^{2}}{2}(1-2 \ln a),
$$

where the two largest contributions are retained. The integrals on the left-hand side of Eqs. (C16) and (C17) are expanded with respect to small $\hbar$ and large $x$ which gives the following two leading contributions:

$$
\int_{x}^{\tilde{x}_{1}} \sqrt{\mathcal{Q}} d x=S\left(x_{1}, x\right)-i \frac{\hbar}{2} \ln \left(\frac{-2 \xi}{P}\right),
$$

when $x \ll x_{1}$, and

$$
\int_{\tilde{x}_{2}}^{x} \sqrt{\mathcal{Q}} d x=S\left(x, x_{2}\right)-i \frac{\hbar}{2} \ln \left(\frac{2 \xi}{P}\right),
$$

when $x \gg x_{2}$. Comparing Eqs. (C18), (C19), and (C20) gives the coordinate transformation

$$
i \frac{z^{2}}{4}-i a^{2} \ln (-z)=S\left(x_{1}, x\right)-i \frac{\hbar}{2} \ln \left(\frac{-2 \xi}{P}\right)+i \hbar \varrho
$$

in the limit $x \ll x_{1}$ and

$$
i \frac{z^{2}}{4}-i a^{2} \ln z=S\left(x, x_{2}\right)-i \frac{\hbar}{2} \ln \left(\frac{2 \xi}{P}\right)+i \hbar \varrho
$$

at $x \gg x_{2}$, where

$$
\begin{aligned}
\varrho & =\frac{a^{2}}{2 \hbar}(1-2 \ln a)+\frac{1}{2}\left(v+\frac{1}{2}\right) \ln \frac{v+1 / 2}{v}-\frac{1}{4} \\
& =\frac{1}{2}\left(v+\frac{1}{2}\right)\{1-\ln (\hbar v)\}-\frac{1}{4} .
\end{aligned}
$$

The transfer matrix in Eq. (19) are obtained in the following steps: (1) taking the asymptotic expansion of solution (C11) at large $z$ and small $\hbar,(2)$ expressing obtained asymptotes as functions of $x$ using Eqs. (C15), (C21), and (C22), (3) constructing asymptotic solution of the original Dirac eigenvalue problem using Eq. (C3) and inverse transformations (C1), and, finally, (4) finding relations for the coefficients of the incoming and outgoing wave solutions. Following this procedure, which involved rather tedious but straightforward calculations, we obtain the coefficients of the transfer matrix in Eq. (19) for pair of turning points $x_{1,2}$. The calculations for the turning points $x_{3,4}$ are done analogously. Finally, we note that the total transfer matrix and the scattering coefficients in Eqs. (23) for the potential barrier are obtained within the approximation, that the propagation between turning points $x_{3}$ and $x_{4}$ is described by the leading order WKB solution $\boldsymbol{\Psi}_{0}$. We note that this approximation generally fails when the applicability conditions of the WKB approximations are not satisfied, in particular when $\left[x_{3}, x_{4}\right]$ is small.
[1] A. K. Geim and K. S. Novoselov, Nat. Mater. 6, 183 (2007).

[2] A. H. Castro Neto, F. Guinea, N. M. R. Peres, A. K. Geim, and K. S. Novoselov, Rev. Mod. Phys. 81, 109 (2009).

[3] N. M. R. Peres, Rev. Mod. Phys. 82, 2673 (2010).

[4] O. Klein, Z. Phys. 53, 157 (1929).

[5] M. I. Katsnelson, K. S. Novoselov, and A. K. Geim, Nat. Phys. 2, 620 (2006).

[6] C. W. J. Beenakker, Rev. Mod. Phys. 80, 1337 (2008).

[7] F. Miao, S. Wijerante, Y. Zhang, U. C. Coskun, W. Bao, and C. N. Lau, Science 317, 1530 (2007).

[8] R. Danneau, F. Wu, M. F. Craciun, S. Russo, M. Y. Tomi, J. Salmilehto, A. F. Morpurgo, and P. J. Hakonen, Phys. Rev. Lett. 100, 196802 (2008).

[9] J. Martin, N. Akerman, G. Ulbricht, T. Lohmann, J. H. Smet, K. von Klitzing, and A. Yacoby, Nat. Phys. 4, 144 (2008).

[10] J. Martin, N. Akerman, G. Ulbricht, T. Lohmann, K. von Klitzing, J. H. Smet, and A. Yacoby, Nat. Phys. 5, 669 (2009).

[11] A. O'Haare, F. V. Kusmartsev, and K. I. Kugel, Nano Lett. 12, 1045 (2012).

[12] P. Sutter, Nat. Mater. 8, 171 (2009); J. Robinson, X. Weng, K. Trumbull, R. Cavalero, M. Wetherington, E. Frantz, M. LaBella, Z. Hughes, M. Fanton, and D. Snyder, ACS Nano 4, 153 (2010).

[13] A. F. Young and P. Kim, Nat. Phys. 5, 222 (2009).

[14] Y.-M. Lin, D. B. Farmer, K. A. Jenkins, W. Yanqing, J. L. Tedesco, R. L. Myers-Ward, C. R. Eddy, D. K. Gaskill,
C. Dimitrakopoulos, and P. Avouris, IEEE Electron. Device Lett. 32, 1343 (2011).

[15] T. Low, F. Guinea, and M. I. Katsnelson, Phys. Rev. B 83, 195436 (2011).

[16] J. C. W. Song, A. V. Shytov, and L. S. Levitov, Phys. Rev. Lett. 111, 266801 (2013).

[17] M. Papagno, S. Rusponi, P. M. Sheverdyaeva, S. Vlaic, M. Etzkorn, D. Pacilé, P. Moras, C. Carbone, and H. Brune, ACS Nano 6, 199 (2012).

[18] S. M. Jung, E. K. Lee, M. Choi, D. Shin, I. Y. Jeon, J. M. Seo, H. Y. Jeong, N. Park, J. H. Oh, and J. B. Baek, Angew. Chem. Int. Ed. 53, 2398 (2014).

[19] Q. Ruge, J. Zheng, G. Luo, Q. Liu, R. Qin, J. Zhou, D. Yu, S. Nagase, W.-N. Mei, Z. Gao, and J. Lu, NPG Asia Mater. 4, e6 (2012).

[20] B. Hunt, J. D. Sanchez-Yamagishi, A. F. Young, M. Yankowitz, B. J. LeRoy, K. Watanabe, T. Taniguchi, P. Moon, M. Koshino, P. Jarillo-Herrero, and R. C. Ashoori, Science 340, 1427 (2013).

[21] C. R. Woods, L. Britnell, A. Eckmann, R. S. Ma, J. C. Lu, H. M. Guo, X. Lin, G. L. Yu, Y. Cao, R. V. Gorbachev, A. V. Kretinin, J. Park, L. A. Ponomarenko, M. I. Katsnelson, Yu. N. Gornostyrev, K. Watanabe, T. Taniguchi, C. Casiraghi, H-J. Gao, A. K. Geim, and K. S. Novoselov, Nat. Phys. 10, 451 (2014).

[22] M. I. Katsnelson, Eur. Phys. J. B 51, 157 (2006). 
[23] J. Tworzydło, B. Trauzettel, M. Titov, A. Rycerz, and C. W. J. Beenakker, Phys. Rev. Lett. 96, 246802 (2006).

[24] B. Sonin, Phys. Rev. B 77, 233408 (2008).

[25] V. V. Cheianov and V. I. Falko, Phys. Rev. B 74, 041403(R) (2006).

[26] A. V. Shytov, M. S. Rudner, and L. S. Levitov, Phys. Rev. Lett. 101, 156804 (2008).

[27] M. V. Fistul and K. B. Efetov, Phys. Rev. Lett. 98, 256803 (2007).

[28] P. G. Silvestrov and K. B. Efetov, Phys. Rev. Lett. 98, 016802 (2007).
[29] T. Tudorovskiy, K. J. A. Reijnders, and M. I. Katsnelson, Phys. Scr. T 146, 014010 (2012).

[30] V. V. Zalipaev, D. N. Maksimov, C. M. Linton, and F. V. Kusmartsev, Phys. Lett. A 377, 216 (2013).

[31] E. B. Sonin, Phys. Rev. B 79, 195438 (2009).

[32] J. M. Pereira, V. Mlinar, F. M. Peeters, and P. Vasilopoulos, Phys. Rev. B 74, 045424 (2006).

[33] M. V. Fedoryuk, Asymptotic Analysis: Linear Ordinary Differential Equations (Springer, Berlin, 1993).

[34] V. P. Maslov, The Complex WKB Method for Nonlinear Equations (Birknäuser, Basel, 1994). 